\title{
Case Report Fatal Morganella morganii bacteraemia in a diabetic
patient with gas gangrene
}

\author{
Correspondence \\ Sujoy Ghosh \\ drsujoyghosh@rediffmail.com
}

Received 17 December 2008

Accepted 16 March 2009

\author{
Sujoy Ghosh, ${ }^{1}$ Abhijit M. Bal, ${ }^{2}$ Iqbal Malik ${ }^{1}$ and Andrew Collier ${ }^{1}$ \\ ${ }^{1}$ Department of Diabetes and Endocrinology, Ayr Hospital, NHS Ayrshire and Arran, Ayr KA6 6DX, \\ Scotland, UK \\ ${ }^{2}$ Department of Medical Microbiology, Ayr Hospital, NHS Ayrshire and Arran, Ayr KA6 6DX, \\ Scotland, UK
}

\begin{abstract}
We report a case of a 60-year-old lady with a history of a heel ulcer that had not responded to antibiotic therapy. This progressed to involve the right leg, which was swollen and erythematous. Radiological imaging revealed the presence of gas within the fascial planes. Blood cultures on admission yielded Morganella morganii. Due to the extent of the gas gangrene and her comorbidities the patient was not suitable for surgical intervention and was treated conservatively with antibiotics. She deteriorated and died within $72 \mathrm{~h}$ of presentation. Non-clostridial gas gangrene is relatively rare, and diagnosis is frequently delayed and often missed. Early aggressive surgical intervention combined with appropriate antibiotic therapy is essential. Bacterial species other than Clostridium should be considered in all cases of gas gangrene.
\end{abstract}

\section{Introduction}

Gas-forming infection, whether clostridial or non-clostridial in origin, is serious and often life-threatening (Bessman \& Wagner, 1975; Bird et al., 1977; Brightmore, 1971). The medical and surgical management of non-clostridial gas gangrene is significantly different from that of clostridial gas gangrene. Non-clostridial gas gangrene is a rare clinical condition and has a high mortality (Darke et al., 1977). Urgent surgical intervention in addition to appropriate antibiotic therapy is essential.

\section{Case report}

A 60-year-old lady was admitted to Ayr Hospital with a 6 week history of a right heel ulcer, presumably from illfitting footwear. She was treated initially by her general practitioner with amoxicillin-clavulanic acid. The patient had a history of poorly controlled insulin-treated type 2 diabetes for the last 16 years. She also had several comorbidities, including metastatic adenocarcinoma of the colon (not amenable to surgery or chemotherapy), atrial fibrillation, hypertension, dyslipidaemia, ischaemic heart disease, stage 3 chronic kidney disease, diabetic retinopathy, peripheral neuropathy, interstitial lung disease, iron deficiency anaemia and osteoporosis. She did not have any evidence of peripheral vascular disease or chronic venous insufficiency. She had no pets at home.

Examination revealed a $2.5 \times 2.5 \mathrm{~cm}$ ulcer on the right heel and a grossly swollen erythematous right leg. The swelling and redness extended up to the right thigh. There was also an area of ulceration $(5 \times 3.5 \mathrm{~cm})$ over the right calf
(Fig. 1). The patient stated that initially she had noticed an area of redness over the calf, which subsequently developed into bullae, before coalescing and subsequently ulcerating. The base of the ulcerated area was necrotic. On palpation of the affected limb there was no crepitus. Systemically the patient was generally unwell. She was febrile, tachycardic, hypotensive and had features of septic shock. Laboratory investigations showed a markedly elevated white cell count $\left(30.9 \times 10^{9}\right.$ cells $\left.^{-1}\right)$, neutrophil count $\left(28.4 \times 10^{9}\right.$ cells $\left.^{-1}\right)$ and C-reactive protein $\left(288 \mathrm{mg} \mathrm{l}^{-1}\right)$. Urea $\left(19.6 \mathrm{mmol}^{-1}\right)$ and creatinine levels $\left(152 \mu \mathrm{mol}^{-1}\right)$ were also raised. Liver function tests were normal prior to admission. There was a mild elevation of bilirubin (32 $\mathrm{U}^{-1}$ ) in keeping with sepsis. There was no evidence of urinary tract infection.

Chest X-ray revealed evidence of long standing interstitial lung disease and electrocardiography revealed evidence of ischaemic heart disease. Radiological imaging revealed the presence of gas within the fascial planes (muscles) of the right leg (Fig. 2). Escherichia coli and Proteus spp. were isolated from superficial swabs. Swabs were not obtained from deeper tissues. Two separate peripheral blood cultures taken on the day of admission yielded a nonlactose fermenting Gram-negative bacillus within $48 \mathrm{~h}$ of incubation. The identity of the organism was established with the help of a VITEK 2 GN colorimetric card that was read with VITEK 2 system software version VT2-R05.01. The probability of the isolate being Morganella morganii was 0.99 . The organism was susceptible to ciprofloxacin, gentamicin, ceftriaxone and meropenem. Because of the possibility of inducible resistance, treatment with meropenem was advised. Clostridium perfringens was not isolated from any of the samples. 


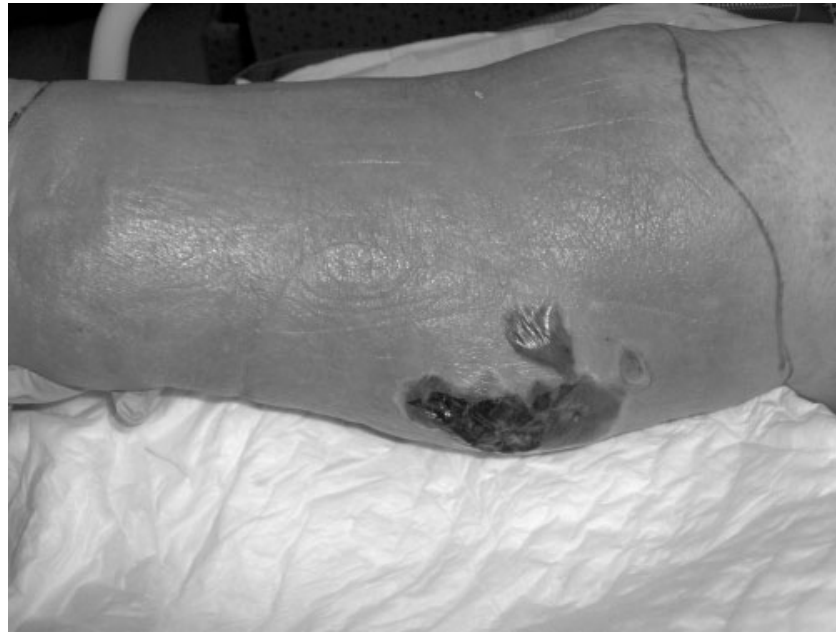

Fig. 1. Clinical image showing the ulcerated area of the right calf.

The patient was referred to the surgical and anaesthetic team. The pros and cons of surgery and general anaesthesia were discussed in detail by the multidisciplinary team and with both the patient and her family. Amputation was considered, but due to her co-morbidities and extent of disease it was felt that the likelihood of her surviving surgery was slim. The patient was managed conservatively with intravenous broad-spectrum antibiotics ( 2 g meropenem three times daily and $400 \mathrm{mg}$ teicoplanin once daily, following an initial loading dose). She was kept comfortable and supportive care was provided. She deteriorated and died within $72 \mathrm{~h}$ of presentation.

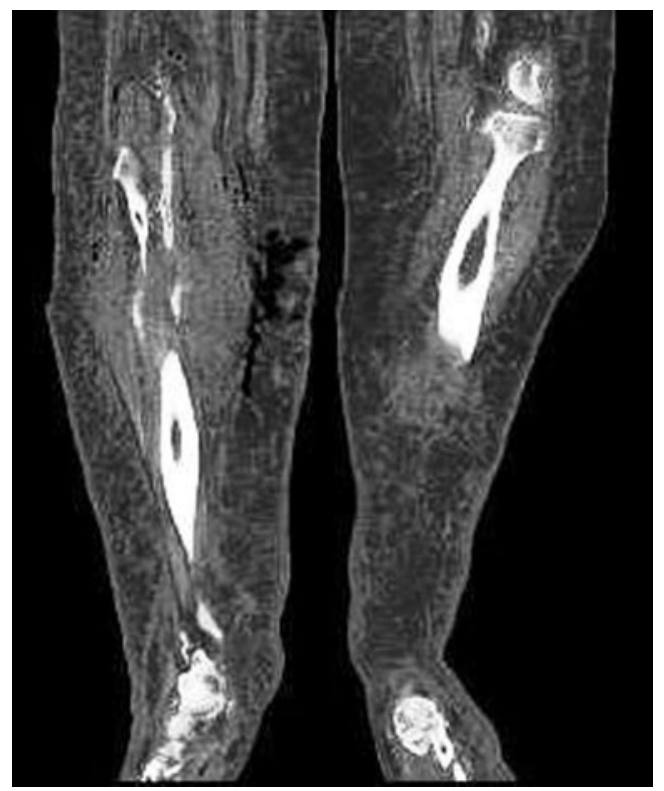

Fig. 2. Computed tomography scan showing the presence of gas within the right leg.

\section{Discussion}

Non-clostridial gas gangrene is relatively rare, and diagnosis is frequently delayed and often missed. Delay in the preliminary diagnosis and failure to undertake urgent aggressive surgical intervention is associated with a poor outcome. An early intervention of this type combined with appropriate antibiotic therapy is essential. Non-clostridial gas gangrene is often associated with trauma (Hubens et al., 1989; Yasuda et al., 1986). However, non-traumatic infection may occur in the absence of cutaneous wounds and tends to be more common in individuals suffering from diabetes mellitus, congestive heart failure, renal failure, nutritional deficiencies, and haematological and gastrointestinal malignancies or any other immunocompromised state. M. morganii is often associated with urinary tract and hepatobiliary infections (McDermott \& Mylotte, 1984). Other risk factors include old age, the presence of concomitant bacteraemia, hospitalization, recent surgery and concurrent antibiotic use (Lee \& Liu, 2006). The progress of non-clostridial gas gangrene is often deceptive with a slow initial phase followed by rapid progression with a risk of septicaemia (Kim et al., 2007). Specific symptoms like local pain and swelling are less marked than that of clostridial gas gangrene. This initial lack of definite symptoms often leads to a delayed diagnosis and a poor outcome.

Non-clostridial gas gangrene is usually caused by a mixed infection, including infection by bacteria such as: Enterococcus faecalis, Escherichia coli, Staphylococcus aureus, group G streptococcus, Klebsiella pneumoniae, Proteus vulgaris and Citrobacter diversus. M. morganii bacteraemia has not previously been reported from a patient with nonclostridial gas gangrene. However, Morganella has been isolated from a patient with polymicrobial non-clostridial myonecrosis (Takahira et al., 2002). There is only one report of isolation of M. morganii from a sacral pressure ulcer as part of a mixed pyogenic infection (Setsuko et al., 2006).

In our patient, coliforms were isolated from superficial leg swabs, which could reflect polymicrobial sepsis. Polymicrobial infection plays an important role in pathogenesis. Facultative anaerobes such as coliforms reduce the redox potential in tissues thereby allowing obligate anaerobes to multiply. Anaerobes on the other hand inhibit phagocytosis (Styrt \& Gorbach, 1989).

Morganella belongs to the family Enterobacteriaceae. It is present in the environment and is part of the normal flora of the colon. It is a motile Gram-negative bacillus and ferments glucose with the production of acid and gas (Janda et al., 1996). Fermentation of glucose in tissues is thought to be the source of the gas in gangrenous infections (Chi et al., 1995). M. morganii bacteraemia is relatively uncommon but has a high rate of mortality. Urinary tract infection is probably the most common infection caused by $M$. morganii in humans, but the organism has also been documented as causing pneu- 
monia, empyema, wound infection, pericarditis, peritonitis, sepsis, arthropathy, endophthalmitis, meningitis, ecthyma gangrenosum-like eruptions and Fournier's gangrene (Bagel \& Grossman, 1985; Cafferkey et al., 1988; Cunningham et al., 1997; Del Poso et al., 1998; Isobe et al., 1994; Mastroianni et al., 1994; Sica et al., 1995; García Reinoso et al., 1990).

It is well documented that patients with colonic cancer are more likely to develop infective endocarditis due to Streptococcus bovis. M. morganii is part of the normal flora of the colon and our patient had an inoperable adenocarcinoma of the colon. It is not clear if this was related to the development of Morganella bacteraemia. However, bacteraemia followed by secondary seeding of a compromised tissue might have led to gangrenous infection.

Production of extended spectrum $\beta$-lactamases and induction of $\beta$-lactamases upon therapy has been widely reported in Morganella (Choi et al., 2008). Carbapenems are therefore considered as the first-line antibiotics for the treatment of life-threatening infections due to Morganella. Unlike clostridial gas-gangrene, hyperbaric oxygen therapy in non-clostridial gas gangrene in diabetics is ineffective (Owada et al., 1994).

In summary, bacterial species other than Clostridium should be considered in all cases of gas gangrene. Antibiotic therapy should be directed towards polymicrobial sepsis because of the life-threatening nature of this condition.

\section{References}

Bagel, J. \& Grossman, M. E. (1985). Hemorrhagic bullae associated with Morganella morganii septicemia. J Am Acad Dermatol 12, 575576.

Bessman, A. N. \& Wagner, W. (1975). Nonclostridial gas gangrene. Report of 48 cases and review of the literature. JAMA 233, 958-963.

Bird, D., Giddings, A. E. \& Jones, S. M. (1977). Non-clostridial gas gangrene in the diabetic lower limb. Diabetologia 13, 373-376.

Brightmore, T. (1971). Non-clostridial gas infection. Proc R Soc Med 64, 1084-1085.

Cafferkey, M. T., Buckley, T. F. \& O'Morain, C. A. (1988). Association of reactive arthropathy and Morganella morganii cross reacting with Yersinia enterocolitica. Scand J Infect Dis 20, 439-442.

Chi, C. H., Chen, K. W., Huang, J. J., Chuang, Y. C. \& Wu, M. H. (1995). Gas composition in Clostridium septicum gas gangrene. J Formos Med Assoc 94, 757-759.

Choi, S. H., Lee, J. E., Park, S. J., Choi, S. H., Lee, S. O., Jeong, J. Y., Kim, M. N., Woo, J. H. \& Kim, Y. S. (2008). Emergence of antibiotic resistance during therapy for infections caused by Enterobacteriaceae producing AmpC $\beta$-lactamase: implications for antibiotic use. Antimicrob Agents Chemother 52, 995-1000.
Cunningham, E. T., Jr, Whitcher, J. P. \& Kim, R. Y. (1997). Morganella morganii postoperative endophthalmitis. Br J Ophthalmol 81, 170171.

Darke, S. G., King, A. M. \& Slack, W. K. (1977). Gas gangrene and related infection: classification, clinical features and aetiology, management and mortality. A report of 88 cases. Br J Surg 64, 104112.

Del Poso, J., Garcia-Silva, J., Almagro, M., Martinez, W., Nicolas, R. \& Fonseca, E. (1998). Ecthyma gangrenosum-like eruption associated with Morganella morganii infection. Br J Dermatol 139, 520-521.

García Reinoso, C., Gómez Rubio, M., Sáez-Royela, F. \& Melero Calleja, E. (1990). Fournier's disease: a report of 9 cases. Rev Esp Enferm Dig 78, 131-134 in Spanish.

Hubens, G., Carly, B., De Boeck, H., Vansteenland, H. \& Wylock, P. (1989). "Spontaneous" non clostridial gas gangrene: case report and review of the literature. Acta Chir Belg 89, 25-28.

Isobe, H., Motomura, K., Kotou, K., Sakai, H., Satoh, M. \& Nawata, H. (1994). Spontaneous bacterial empyema and peritonitis caused by Morganella morganii. J Clin Gastroenterol 18, 87-88.

Janda, J. M., Abbott, S. L., Khashe, S. \& Robin, T. (1996). Biochemical investigations of biogroups and subspecies of Morganella morganii. $J$ Clin Microbiol 34, 108-113.

Kim, J. H., Cho, C. R., Um, T. H., Rhu, J. Y., Kim, E. S., Jeong, J. W. \& Lee, H. R. (2007). Morganella morganii sepsis with massive hemolysis. J Korean Med Sci 22, 1082-1084.

Lee, I. K. \& Liu, J. W. (2006). Clinical characteristics and risk factors for mortality in Morganella morganii bacteremia. J Microbiol Immunol Infect 39, 328-334.

Mastroianni, A., Coronado, O. \& Chiodo, F. (1994). Morganella morganii meningitis in a patient with AIDS. J Infect 29, 356-357.

McDermott, C. \& Mylotte, J. M. (1984). Morganella morganii: epidemiology of bacteremic disease. Infect Control 5, 131-137.

Owada, R., Kanamori, A., Hirai, N. \& Yajima, Y. (1994). A case of NIDDM with non-clostridial gas-producing infection in the lower limb - the effects of hyperbaric oxygen therapy. Kansenshogaku Zasshi 68, 263-267 (in Japanese).

Setsuko, T., Masaaki, T. \& Osamu, I. (2006). Two cases of nonclostridial gas gangrene developed from sacral pressure ulcer. Jpn J Press Ulcers 8, 188-194.

Sica, S., Di Mario, A., Salutari, P., d'Onofrio, G., Antinori, A., Chiusolo, P. \& Leone, G. (1995). Morganella morganii pericarditis after resolvent splenectomy for immune pancytopenia following allogeneic bone marrow transplantation for acute lymphoblastic leukemia. Clin Infect Dis 21, 1052-1053.

Styrt, B. \& Gorbach, S. L. (1989). Recent developments in the understanding of the pathogenesis and treatment of anaerobic infections (2). $N$ Engl J Med 321, 240-246.

Takahira, N., Shindo, M., Tanaka, K., Soma, K., Ohwada, T. \& Itoman, M. (2002). Treatment outcome of nonclostridial gas gangrene at a level 1 trauma center. J Orthop Trauma 16, 12-17.

Yasuda, K., Hayashi, M., Takeda, N., Goshima, E. \& Miura, K. (1986). A survived case of diabetic nonclostridial gas gangrene and the review of the literatures on microbiological findings. Jpn J Med 25, 171-174. 